\title{
An Efficient Block based Feature Level Image Fusion Technique using Wavelet Transform and Neural Network
}

\author{
C.M.Sheela Rani \\ Research Scholar \\ Acharya Nagarjuna University, \\ Guntur, AP, India
}

\author{
V.VijayaKumar \\ Phd, Dept of Computers, Head \\ SRRF, GIET, JNTUK \\ Rajahmundry, AP, India
}

\author{
B.Sujatha \\ Assoc. Professor in CSE \\ GIET, JNTUK \\ Rajahmundry, AP, India
}

\begin{abstract}
Image fusion is a process of combining relevant information from two or more images into a single informative image. In this paper, wavelet transform is integrated with neural network, which is one of the feature extraction or detection machine learning applications. This paper has derived an efficient block based feature level wavelet transform with neural network (BFWN) model for image fusion. In the proposed BFWN model, the two fusion techniques, discrete wavelet transform (DWT) and neural network (NN) are discussed for fusing IRS-1D images using LISS III scanner about the location Hyderabad, Vishakhapatnam, Mahaboobnagar and Patancheru in India. Also QuickBird image data and Landsat 7 image data are used to perform experiments on the proposed BFWN method. The features under study are contrast visibility, spatial frequency, energy of gradient, variance and edge information. Feed forward back propagation neural network is trained and tested for classification since the learning capability of neural network makes it feasible to customize the image fusion process. The trained neural network is then used to fuse the pair of source images. The proposed BFWN model is compared with DWT alone to assess the quality of the fused image. Experimental results clearly prove that the proposed BFWN model is an efficient and feasible algorithm for image fusion.
\end{abstract}

\section{General Terms}

Discrete wavelet transform, neural network

\section{Keywords}

Image fusion, DWT, Neural Network, block based features, performance measures.

\section{INTRODUCTION}

The concept of image fusion has been used in many applications like remote sensing, medicine, automatic change detection, biometrics etc. To acquire an image with all the relevant and necessary information is not possible by using image-capturing devices. Hence, there is a need to fuse multiple images. Image fusion mainly focuses on combining spatial information of a high resolution Panchromatic (PAN) image and spectral information of a low resolution Multispectral image (MS) to produce an image with highest spatial content while preserving spectral resolution. The technique of image fusion helps to obtain an image with all the relevant information. Depending upon the purpose of fusion, the fusion techniques can be classified into five groups [1]. Fusion of multi-view images, multimodal images, multitemporal images, multifocus images and fusion for image restoration. Multiview image fusion integrates the images from the same modality and taken at the same time but from different viewpoints. Multimodal image fusion integrates the images taken by different sensors to arrive at conclusions that are not feasible from a single sensor. Multitemporal fusion combines the images taken at different times in order to detect changes between them or to synthesize realistic images of objects which were not photographed in a desired time. Multifocus fusion of images deals with 3D scene taken repeatedly with various focal lengths. Ishita De and Bhabatosh Chanda proposed an algorithm for fusing multifocus images using morphological wavelets [2]. The multisensor image fusion helps in understanding the existing surroundings in a better way, which leads in better planning, decision-making, and control of autonomous and intelligent machines [3].

The fusion process can be performed at different levels of information representation such as pixel level, feature level and symbolic level. In pixel-level image fusion, the simple mathematical operations such as averaging, addition, subtraction/omission of the pixel intensities are applied on the pixel values of the source images. Generally, these methods have some side effects such as reducing the contrast of the image, etc. The next level operates on the characteristics of the image such as size, shape, edge, etc. This type of fusion comes under feature level multi-focus image fusion. Siddiqui et al. proposed an algorithm for block-based feature-level multi-focus image fusion in [4]. G. Piella proposed a regionbased multi-resolution image fusion algorithm which combines the aspects of region and pixel-based fusion [5]. The highest level of fusion is decision level fusion which deals with symbolic representation of images [6]. Currently, most of the image fusion has been performed using pixel based methods [7], [8].

In addition to simple pixel-level image fusion techniques, complex techniques such as Laplacian Pyramid, Discrete Wavelet Transform, Neural Network and advance Discrete Wavelet Transform techniques have been proposed. All these techniques have their own merits and demerits. Hence, the fusion techniques range from the simplest method of pixel averaging to more sophisticated method which integrates multi-resolution technique with neural network. A. Toet proposed an algorithm for image fusion using ratio of low pass pyramid [9]. K. Kannan et al. evaluated the performance of all the levels of multi-focused images using different wavelet transforms [10]. Dong et al. discussed various advances in multi-sensor data fusion [11]. Vijayaraj provided the concepts of image fusion in remote sensing applications [12].

This paper is organized into six sections. The section 2 describes image fusion based on wavelet transform; section 3 describes about artificial neural networks and the proposed 
BFWN algorithm; section 4 deals with quality assessment techniques; section 5 deals with results and discussions; conclusions in section 6 .

\section{IMAGE FUSION BASED ON WAVELET TRANSFORM}

Image fusion combines the information from different sensors taken from the same scene to achieve a new fused image, which contains the best information coming from the source images. Hence, the fused image will have a better quality than any of the original source images. Shi et al. presented a multiband wavelet-based image fusion method, which is a further development of the two-band wavelet transform [13]. Yao et al. proposed a new weighting technique based on wavelet transform for the fusion of a high spatial resolution PAN image and a low spatial resolution MS image and also proposed a multi-spectral image fusion algorithm based on wavelet transform characteristic of human vision system [14].

The wavelet-based approach is appropriate for performing fusion task for the following reasons:

- It is a multiscale approach which well suits to manage the different image resolutions. The multiscale information can be useful in a number of image processing applications including image fusion.

- It allows image decomposition in different kinds of coefficients preserving the image information. These coefficients can be appropriately combined to obtain new coefficients so that the information in the source images is collected appropriately.

- The final fused image is achieved by simply performing inverse wavelet transform.
The wavelet transform decomposes an image into four frequency bands: low-low (LL), low-high (LH), high-low (HL) and high-high $(\mathrm{HH})$. It provides a framework in which an image is decomposed, with each level corresponding to a coarser resolution band. For example, for fusing a MS image with a high-resolution PAN image using wavelet fusion technique, the PAN image is first decomposed into a set of low-resolution PAN images with corresponding wavelet coefficients for each level. An individual band of the MS image then replaces the low-resolution PAN at the resolution level of the original MS image. The high resolution spatial information is injected into each MS band by performing an inverse wavelet transform on each MS band together with the corresponding wavelet coefficients. In the wavelet-based fusion schemes, detailed information is extracted from the PAN image using wavelet transforms and injected into the MS image. This entire process is represented in Figure 1.

\section{ARTIFICIAL NEURAL NETWORKS}

The Artificial Neural Network (ANN) based method employs a nonlinear response function that iterates many times in a special network structure in order to learn the complex functional relationship between the input and output training data. A neural network consists of three layers - input layer, hidden layer and output layer. The input layer contains many neurons, which represent the feature factors extracted and normalized from the source images. The hidden layer consists of several neurons for processing and the output layer can have one or more neurons. In general, the $\mathrm{i}^{\text {th }}$ neuron of the input layer connects with the $\mathrm{j}^{\text {th }}$ neuron of the hidden layer by some specified weight and the $\mathrm{j}^{\text {th }}$ neuron of the hidden layer connects with the $\mathrm{k}^{\mathrm{th}}$ neuron of output layer by some specified weight. The weighting function is used to simulate and recognize the response relationship between the features of fused image and corresponding features from the source images.

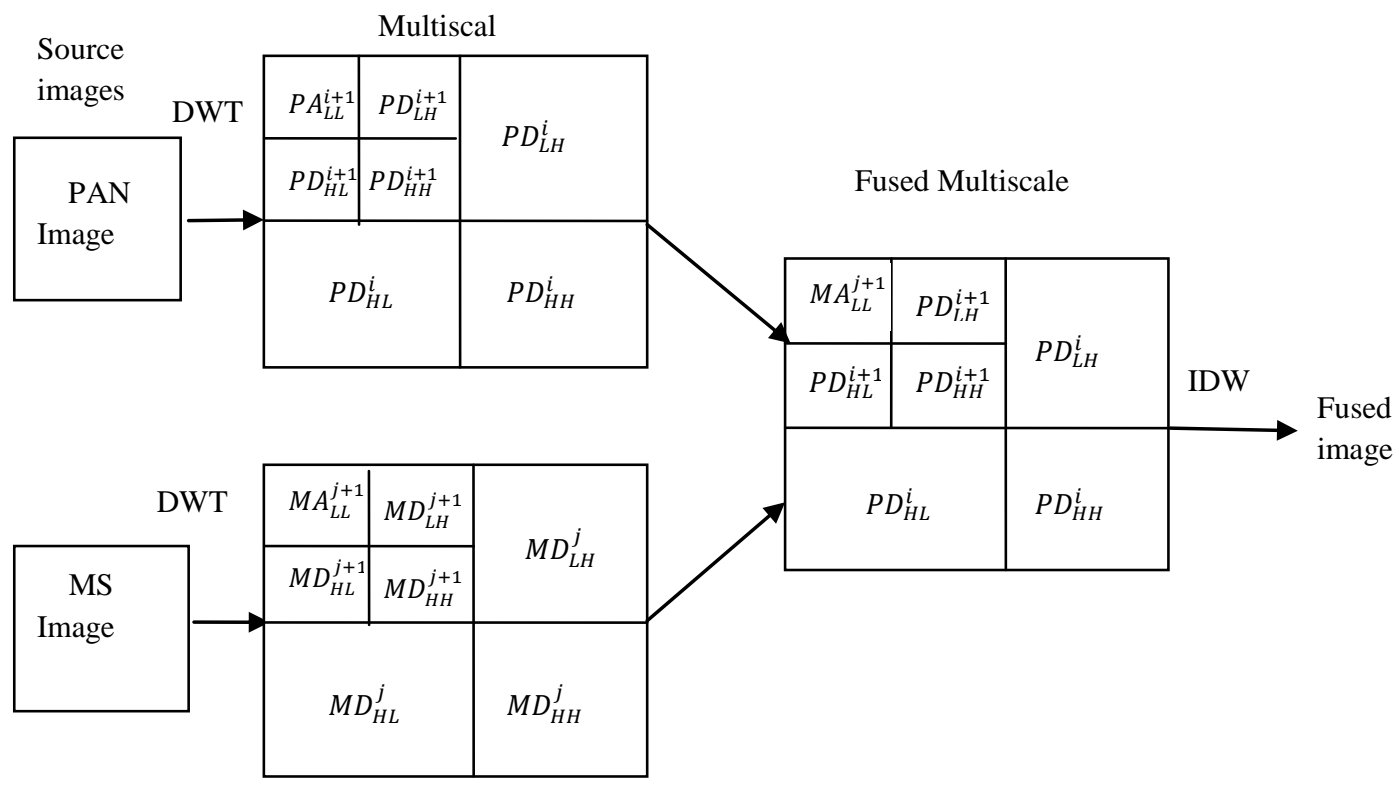

Fig 1: Block diagram of the fusion scheme using DWT.

ANN is more powerful and self-adaptive technique for applications such as pattern recognition when compared to traditional linear and simple nonlinear analysis [15], [16]. Li et al. describes the application of ANN to pixel-level fusion of multi-focus images taken from the same scene [17].
Sahoolizadeh et al. proposed a new hybrid approach for face recognition using Gabor Wavelets and Neural Networks [18]. Rong et al. presented a feature-level image fusion method based on segmentation region and neural networks [19]. 
The first step of neural network based data fusion is to decompose the two registered images into several blocks with some pre-defined size. The features are extracted from the two images of the corresponding blocks, and the normalized feature vector incident to the neural network is constructed. Generally, the features used for extraction are spatial frequency, visibility, edge, etc. Next, the NN is trained by selecting a sample vector. After training, the NN model can remember a functional relationship and can be used for further calculations. For these reasons, the $\mathrm{NN}$ concept is adopted to develop nonlinear models for multiple sensor image fusion. The learning capability of NN makes it feasible to customize the image fusion process. Many applications indicate that, the ANNbased fusion methods have many advantages, especially when input multiple sensor data were incomplete or with much noise.

\subsection{Features Selection}

In feature-level image fusion, the selection of different features is an important task. The five different features used to characterize the information level contained in a specific portion of the image are Contrast Visibility, Spatial Frequency, Variance, Energy of Gradient (EOG), and Edge information. R.Maruthi et al. proposed a fusion procedure by using a selection mode according to the magnitude of the spatial frequency and Visibility [20].

Contrast Visibility: Contrast visibility relates to the clearness level of the block. It calculates the deviation of a block of pixels from its block's mean value. The visibility of the image block is obtained using equation (1).

$$
V I=\frac{1}{p * q} \sum_{m, n \in B_{k}} \frac{\left|I(m, n)-\mu_{k}\right|}{\mu_{k}}
$$

where $\mu_{\mathrm{k}}$ and $\mathrm{p} * \mathrm{q}$ are the mean and size of the block $B_{k}$ respectively.

Spatial Frequency: Spatial frequency measures the activity level in an image. It is used to calculate the frequency changes along rows and columns of the image. It is measured using equations (2), (3) and (4).

$$
\mathrm{SF}=\sqrt{(\mathrm{RF})^{2}+(\mathrm{CF})^{2}}
$$

Where $\quad \mathrm{RF}=\sqrt{\frac{1}{\mathrm{p} * \mathrm{q}} \sum_{\mathrm{m}=1}^{\mathrm{p}} \sum_{\mathrm{n}=2}^{\mathrm{q}}[\mathrm{I}(\mathrm{m}, \mathrm{n})-\mathrm{I}(\mathrm{m}, \mathrm{n}-1)]^{2}}$

$$
\mathrm{CF}=\sqrt{\frac{1}{\mathrm{p} * \mathrm{q}} \sum_{\mathrm{n}=1}^{\mathrm{q}} \sum_{\mathrm{m}=2}^{\mathrm{p}}[\mathrm{I}(\mathrm{m}, \mathrm{n})-\mathrm{I}(\mathrm{m}-1, \mathrm{n})]^{2}}
$$

where I is the image and $\mathrm{p}^{*} \mathrm{q}$ is the image size. A large value of spatial frequency describes good information level in the image and therefore it measures the clearness of the image.

Variance: Variance is used to measure the extent of focus in an image block. It is calculated using equation (5).

$$
\text { Variance }=\frac{1}{\mathrm{p} * \mathrm{q}} \sum_{\mathrm{m}=1}^{\mathrm{p}} \sum_{\mathrm{n}=1}^{\mathrm{q}}(\mathrm{I}(\mathrm{m}, \mathrm{n})-\mu)^{2}
$$

where $\mu$ is the mean value of the block image and $p^{*} q$ is the image size. A high value of variance shows the greater extent of focus in the image block.
Energy of Gradient (EOG): EOG is used to measure the amount of focus in an image. It is calculated using equations (6), (7) and (8).

$$
\begin{aligned}
& \text { EOF }=\sum_{m=1}^{\mathrm{p}-1} \sum_{\mathrm{n}=1}^{\mathrm{q}-1}\left(\mathrm{a}_{\mathrm{m}}^{2}+\mathrm{a}_{\mathrm{n}}^{2}\right) \\
& \text { where } \quad \mathrm{a}_{\mathrm{m}}=\mathrm{a}(\mathrm{m}+1, \mathrm{n})-\mathrm{a}(\mathrm{m}, \mathrm{n}) \\
& \text { and } \quad \mathrm{a}_{\mathrm{n}}=\mathrm{a}(\mathrm{m}, \mathrm{n}+1)-\mathrm{a}(\mathrm{m}, \mathrm{n})
\end{aligned}
$$

where $\mathrm{p}$ and $\mathrm{q}$ represent the dimensions of the image block. A high value of energy of gradient shows greater amount of focus in the image block.

Edge Information: The Canny edge detector is used to identify the edge pixels in the image block. It returns 1 if the current pixel belongs to some edge in the image otherwise it returns 0 . The edge feature is just the number of edge pixels contained within the image block.

\subsection{Proposed BFWN algorithm}

The proposed block based feature level algorithm which integrates wavelet transform with neural networks is discussed clearly below.

\section{Step.1 Read PAN and MS image.}

Step.2 Apply second level discrete wavelet transform to both the images as shown in Figure 2.

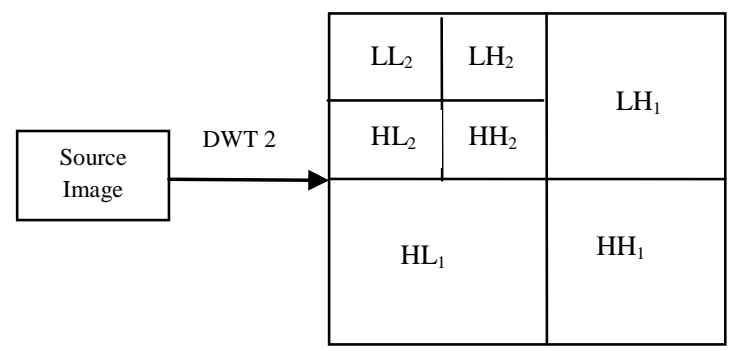

Fig. 2 Second level decomposition

Step. 3 Consider the $\mathrm{LL}_{2}$ component of both the images.

Step. 4 Partition the $\mathrm{LL}_{2}$ component of both the images with into $\mathrm{k}$ blocks of $\mathrm{M} \times \mathrm{N}$ size and extract the features for every block. The features under study are contrast visibility, spatial frequency, energy of gradient, variance and edge information.

Step. 5 Subtract the feature values of the $\mathrm{j}^{\text {th }}$ block of $\mathrm{LL}_{2}$ subband of PAN from the corresponding feature values of the $\mathrm{j}^{\text {th }}$ block of $\mathrm{LL}_{2}$ subband of MS. If the difference is 0 then denote it as 1 else -1 .

Step. 6 Construct an Index vector for classification which will be given as an input for the neural network.

Step. 7 Create a neural network with adequate number of layers and neurons. Train the newly constructed neural network with random index value.

Step. 8 Simulate the neural network with feature vector index value. 
Step. 9 If the simulated output $>1$ then the $\mathrm{j}^{\text {th }}$ subblock of $\mathrm{LL}_{2}$ subband of PAN image is considered else the $\mathrm{j}^{\text {th }}$ subblock of $\mathrm{LL}_{2}$ subband of MS image is considered.

Step. 10 Reconstruct the entire block and apply inverse wavelet transform to get back the fused image.

The block diagram of the proposed BFWN method is shown in Figure 3 .

\section{QUALITY ASSESSMENT TECHNIQUES}

To assess the quality of fused image, some quality measures are required. Goal of image quality assessment is to supply quality metrics that can predict perceived image quality automatically. While visual inspection has limitation due to human judgment, quantitative approach based on the evaluation of "distortion" in the resulting fused image is more desirable for mathematical modeling.

\subsection{Qualitative Measures}

The goals of the quantitative measures are normally used for the result of visual inspection due to the limitations of human eyes. In Mathematical modeling, quantitative measure is desirable. One can develop quantitative measures to predict perceived image quality. In this work, the quality assessment is derived on BFWN method using noise-based measures to evaluate the noise of the fused image compared to the original MS image. The following optimal noise-based measures are implemented to judge the performance of fusion methods [21] as follows:

Peak Signal to Noise Ratio (PSNR): PSNR is used to reveal the radiometric distortion of the fused image compared to the original image. It is defined using equation (9).

$$
\operatorname{PSNR}(d B)=10 \log _{10}\left(\frac{255 * 255}{\mathrm{MSE}}\right)
$$

where MSE is used to measure the spectral distortion in the fused image. It is defined using equation (10)

$$
\operatorname{MSE}=\frac{\sum_{\mathrm{i}=1}^{\mathrm{M}} \sum_{\mathrm{j}=1}^{\mathrm{N}}\left(\mathrm{I}_{\mathrm{R}}(\mathrm{i}, \mathrm{j})-\mathrm{I}_{\mathrm{F}}(\mathrm{i}, \mathrm{j})^{2}\right)}{\mathrm{M} * \mathrm{~N}}
$$

.where $I_{R}(i, j)$ denotes pixel $(i, j)$ of the image reference and $I_{F}(i, j)$ denotes pixel $(i, j)$ of the fused image, $M * N$ is the image size
Mutual Information Measure (MIM): MIM is used to furnish the amount of information of the source image in the used image. It is defined using equation (11).

$$
I_{M N}=\sum_{x, y} P_{M N}(x, y) \log \frac{P_{M N}(x, y)}{P_{M}(x) P_{N}(y)}
$$

where $\mathrm{M}, \mathrm{N}$ are the two source images, $\mathrm{P}_{\mathrm{M}}(\mathrm{x})$ and $\mathrm{P}_{\mathrm{N}}(\mathrm{y})$ are the probability density functions of the individual images and $\mathrm{P}_{\mathrm{MN}}(\mathrm{x}, \mathrm{y})$ is joint probability density function.

Fusion Factor (FF): $\mathrm{FF}$ is used to measure the amount of information present in the fused image. It is defined using equation (12).

$$
\mathrm{FF}=\mathrm{I}_{\mathrm{AF}}+\mathrm{I}_{\mathrm{BF}}
$$

where $\mathrm{A}$ and $\mathrm{B}$ are the two source images and $\mathrm{F}$ is the fused image. A higher value of FF indicates that fused image contains moderately good amount of information present in both the images.

Standard Deviation (SD): SD is used to measure the level of contrast in the fused image. It is defined using equation (13).

$$
S D=\sqrt{\sum_{i=0}^{L}\left(i-i^{\prime}\right)^{2} h_{F}(i)}
$$

where $i^{\prime}=\sum_{i=0}^{L} i h_{F}$ and $h_{F}$ is the normalized histogram of the fused image and $\mathrm{L}$ is the number of gray levels. A well contrast image has high standard deviation.

Mean Absolute Error (MAE): MAE is used to measure the average magnitude of the errors in a set of forecasts, without considering their direction. It measures the accuracy for continuous variables using equation (14).

$$
M A E=\frac{1}{M * N} \sum_{x=0}^{M-1} \sum_{y=0}^{N-1}\left(I_{R}(x, y)-I_{F}(x, y)\right)
$$

where $I_{R}(x, y)$ denotes pixel $(x, y)$ of the image reference and $I_{F}(x, y)$ denotes pixel $(x, y)$ of the fuse image and $M * N$ is the image size.

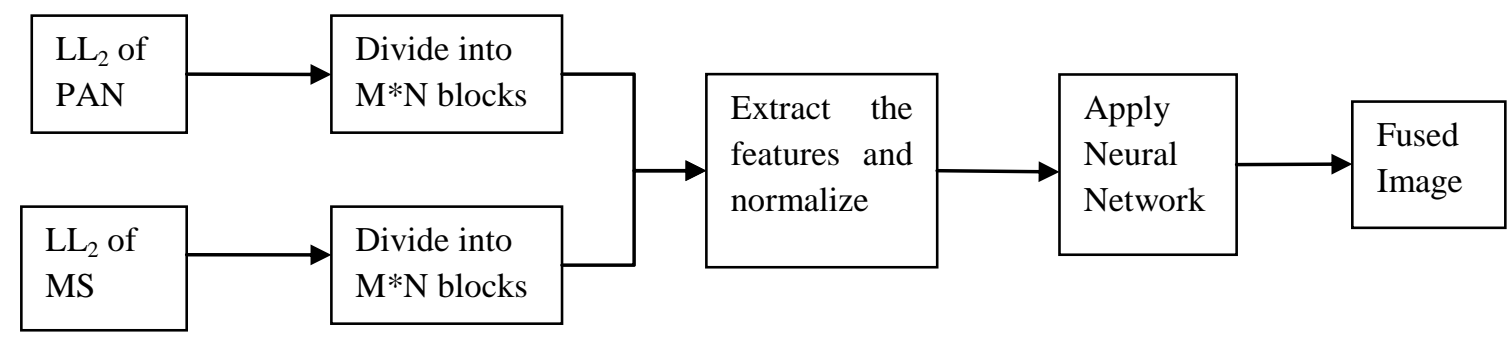

Fig. 3 Block diagram of the proposed method BFWN 


\section{RESULTS AND DISCUSSIONS}

In this paper, two kinds of fusion methods are implemented and executed on PC with 2.4 G CPU and 2.0 G RAM using

Matlab 7.6.0 to compare their fusion results. The experiment is conducted and tested on IRS-1D PAN and MS images for the locations Hyderabad, Vishakhapatnam, Mahaboobnagar and Patancheru in India and on Landsat 7 image data and QuickBird image data.

The following Figure 4 shows the PAN, MS and the fused images about the location Hyderabad by using the methods DWT and BFWN.

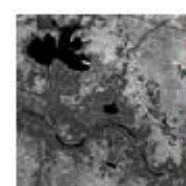

(a)

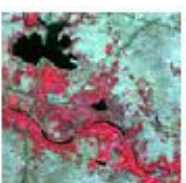

(b)

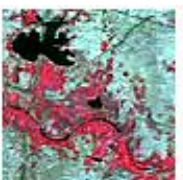

(c)

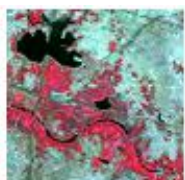

(d)
Fig. 4 (a) PAN image (b) MS image (C) fused image using DWT (d) fused image using BFWN

The following Figure 5 shows the PAN, MS and the fused images about the location Vishakhapatnam by using the methods DWT and BFWN.

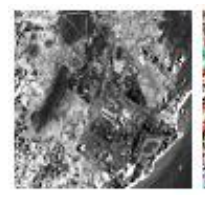

(a)

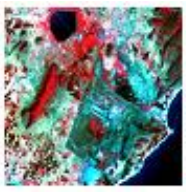

(b)

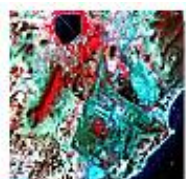

(c)

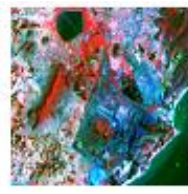

(d)
Fig. 5 (a) PAN image (b) MS image (C) fused image using DWT (d) fused image using BFWN

The following Figure 6 shows the PAN, MS and the fused images about the location Mahaboobnagar by using the methods DWT and BFWN.

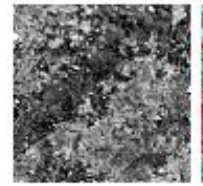

(a)

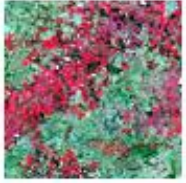

(b)

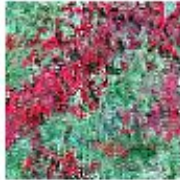

(c)

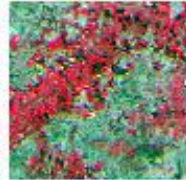

(d)
Fig. 6 (a) PAN image (b) MS image (C) fused image using DWT (d) fused image using BFWN
The following Figure 7 shows the PAN, MS and the fused images about the location Patancheru by using the methods DWT and BFWN.

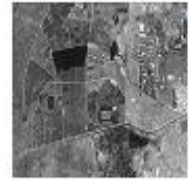

(a)

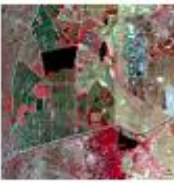

(b)

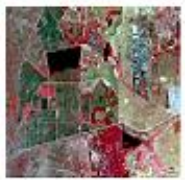

(c)

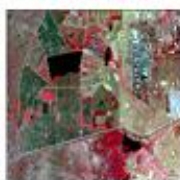

(d)
Fig. 7 (a) PAN image (b) MS image (C) fused image using DWT (d) fused image using BFWN

The following Figure 8 shows the PAN, MS and the fused images of Landsat 7 image data by using the methods DWT and BFWN.

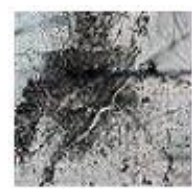

(a)

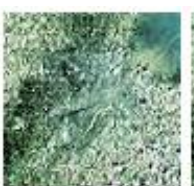

(b)

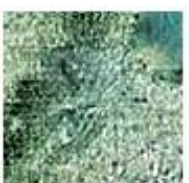

(c)

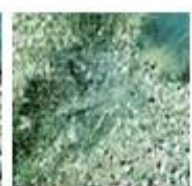

(d)
Fig. 8 (a) PAN image (b) MS image (C) fused image using DWT (d) fused image using BFWN

The following Figure 9 shows the PAN, MS and the fused images of QuickBird image data by using the methods DWT and BFWN.

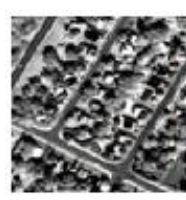

(a)

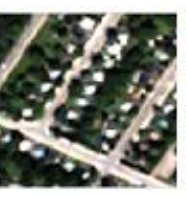

(b)

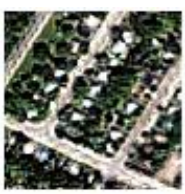

(c)

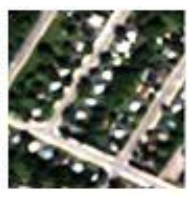

(d)
Fig. 9 (a) PAN image (b) MS image (C) fused image using DWT (d) fused image using BFWN

The calculated quality measures using DWT and BFWN about the location Hyderabad, Visakhapatnam and Mahaboobnagar image data sets are mentioned in table 1 and about the location Patancheru, Landsat 7 and QuickBird image data sets are mentioned in table 2 .

By comparing the values of PSNR for fusing the six pairs of data images using DWT and BFWN, the results show that higher values of PSNR is achieved for the proposed method i.e., BFWN model. The graph is depicted in Figure 10. Similarly, by comparing the values of SD for fusing the six pairs of data images using DWT and BFWN, the results show that smaller values of SD is achieved for the proposed method i.e., BFWN model. The graph is depicted in Figure 11. 
Table 1. Comparison of different metrics using the methods DWT and BFWN for the locations Hyderabad, Visakhapatnam and Mahaboobnagar image data sets.

\begin{tabular}{|c|c|c|c|c|c|c|}
\hline $\begin{array}{c}\text { Qua- } \\
\text { lity } \\
\text { Met- } \\
\text { rics }\end{array}$ & \multicolumn{2}{|c|}{ Hyderabad Image } & \multicolumn{2}{c|}{$\begin{array}{c}\text { Vishakhapatnam } \\
\text { Image }\end{array}$} & \multicolumn{2}{c|}{$\begin{array}{c}\text { Mahaboobnagar } \\
\text { Image }\end{array}$} \\
\cline { 2 - 7 } PSNR & 73.7702 & $\mathbf{7 7 . 0 6 5 8}$ & 70.4177 & $\mathbf{7 0 . 5 0 8 3}$ & 68.7775 & $\mathbf{7 2 . 3 0 1 1}$ \\
\hline MIM & 0.9925 & $\mathbf{2 . 0 2 8 9}$ & 0.9454 & $\mathbf{1 . 0 2 3 5}$ & 0.3762 & $\mathbf{1 . 0 0 6 0}$ \\
\hline FF & 1.9851 & $\mathbf{4 . 0 5 7 7}$ & 1.8908 & $\mathbf{2 . 0 4 7 0}$ & 0.7525 & $\mathbf{2 . 0 1 2 0}$ \\
\hline SD & 44.8092 & $\mathbf{4 0 . 5 1 9 7}$ & 72.1639 & $\mathbf{6 2 . 3 8 7 2}$ & 57.4739 & $\mathbf{4 9 . 4 4 4 0}$ \\
\hline MAE & 0.0235 & $\mathbf{0 . 0 1 6 1}$ & $\mathbf{0 . 0 3 3 5}$ & 0.0357 & 0.0428 & $\mathbf{0 . 0 2 9 1}$ \\
\hline
\end{tabular}

Table 2. Comparison of different metrics using the methods DWT and BFWN for the locations Patancheru, Landsat 7 and QuickBird image data sets.

\begin{tabular}{|c|c|c|c|c|c|c|}
\hline $\begin{array}{c}\text { Qua- } \\
\text { lity } \\
\begin{array}{c}\text { Met- } \\
\text { rics }\end{array}\end{array}$ & \multicolumn{2}{|c|}{$\begin{array}{c}\text { Patancheru } \\
\text { Image }\end{array}$} & \multicolumn{2}{c|}{ Landsat 7 Image } & \multicolumn{2}{c|}{ QuickBird Image } \\
\cline { 2 - 7 } & DWT & BFWN & DWT & BFWN & DWT & BFWN \\
\hline PSNR & 73.7030 & $\mathbf{7 3 . 8 2 6 9}$ & 72.3550 & $\mathbf{7 3 . 4 7 8 9}$ & 70.7147 & $\mathbf{7 5 . 4 5 0 8}$ \\
\hline MIM & 1.0900 & $\mathbf{1 . 1 9 8 5}$ & 0.6365 & $\mathbf{1 . 6 8 4 2}$ & 0.6034 & $\mathbf{1 . 8 4 8 7}$ \\
\hline FF & 2.1800 & $\mathbf{2 . 3 9 7 0}$ & 1.2730 & $\mathbf{3 . 3 6 8 4}$ & 1.2067 & $\mathbf{3 . 6 9 7 4}$ \\
\hline SD & 41.0360 & $\mathbf{3 5 . 4 8 0 4}$ & 41.7186 & $\mathbf{3 9 . 6 2 7 5}$ & 84.0930 & $\mathbf{7 7 . 5 7 1 3}$ \\
\hline MAE & $\mathbf{0 . 0 2 3 3}$ & 0.0258 & 0.0308 & $\mathbf{0 . 0 2 9 6}$ & 0.0331 & $\mathbf{0 . 0 1 9 4}$ \\
\hline
\end{tabular}

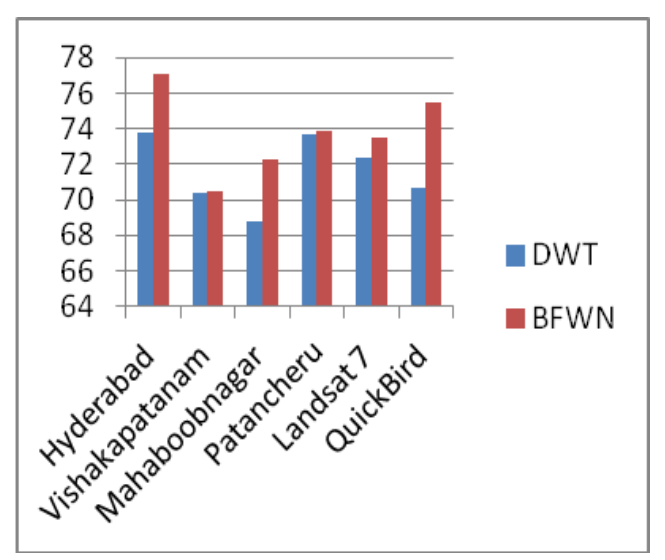

Fig. 10. Comparing PSNR values for DWT and BFWN

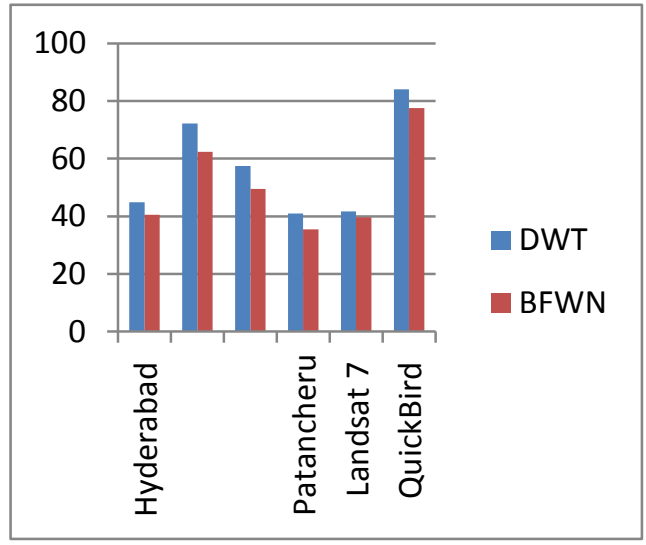

Fig. 11. Comparing SD values for DWT and BFWN

\section{CONCLUSIONS}

The potentials of image fusion using the proposed method BFWN are explored. The various fusion results are analyzed by using quality performance metrics. For all the six image data sets, the higher value for PSNR, MIM and FF is achieved for the proposed BFWN method. The higher value of PSNR implies that, the spectral information in MS image is preserved effectively and high signal is preserved in the fused image. The higher value of MIM and FF indicate that symmetry is achieved by retaining spectral information. The higher value of FF indicates that the fused image contains moderately good amount of information present in both the images. For all the six image data sets, the smaller value for $\mathrm{SD}$ is obtained for the proposed BFWN method. The smaller value of SD indicates that not much deviation is induced in the fused image. Among six images, the smaller value of MAE is achieved for four images by using the proposed BFWN method. The smaller value of MAE indicates that error rate is reduced in the fused image. The metric parameters PSNR, MIM and FF are maximum for the proposed BFWN model, and SD and MAE are minimum for the proposed BFWN model. Hence, it is ascertained that BFWN model has superior performance than wavelet transform alone. The results are verified for LISS III images and the study can be extended to other types of images.

\section{ACKNOWLEDGMENTS}

The authors wish to thank anonymous referees for their constructive comments. The authors would like to express their gratitude to Sri K.V.V.Satyanarayanan Raju, Founder \& Chairman and Sri K.Sasi Kiran Varma, Managing Director, Chaitanya group of Institutions for providing necessary infrastructure to carry out the research work at SRRF in GIET, Rajahmundry.

\section{REFERENCES}

[1] Jan Flusser, Filip Sroubek, and Barbara Zitova, Image Fusion: Principles, Methods, and Applications, Tutorial EUSIPCO 2007 Lecture Notes.

[2] Ishita De and Bhabatosh Chanda, "A simple and efficient algorithm for multifocus image fusion using morphological wavelets" in Signal Processing. pp. 924936, 2006.

[3] Hall, L.; Llinas, J, "An introduction to multisensor data fusion”, Proc. IEEE. 1997, 85, 6-23. 
[4] Abdul Basit Siddiqui, M.Arfan Jaffar, Ayyaz Hussain, Anwar M. Mirza, "Block-based Feature-level Multifocus Image Fusion", 5th International Conference on Future Information Technology (FutureTech), 2010 IEEE, 978-1-4244-6949-9/10.

[5] G. Piella, "A region-based multiresolution image fusion algorithm", Information Fusion, 2002. Proceedings of the Fifth International Conference on Information Fusion, 1557 - 1564 vol.2

[6] F. Sroubek, S. Gabarda, R. Redondo, S.Fischer and G. Crist'obal, "Multifocus Fusion with Oriented Windows", Academy of Sciences, Pod vod'arenskou viejz'ý 4, Prague, Czech Republic; Instituto de 'Optica, CSIC, Serrano 121, 28006 Madrid, Spain.

[7] Zhenhua Li; Zhongliang Jing; Gang Liu; Shaoyuan Sun; Henry Leung, "Pixel visibility based multifocus image fusion" Neural Networks and Signal Processing, 2003.Proceedings of the 2003 International Conference on Volume 2, Issue, 14-17 Dec. 2003 Page(s): 1050 $1053 \mathrm{Vol} .2$

[8] Shutao Li, James T. Kwok and Yaonan Wanga, "Combination of images with diverse focuses using the spatial frequency", Information Fusion, Volume 2, Issue 3, September 2001.

[9] A. Toet, "Image fusion by a ratio of low pass pyramid" in Pattern Recognition Letters, vol. 9, no. 4, pp. 245-253, 1989.

[10] K. Kannan, S. Arumuga Perumal, K. Arulmozhi, "Performance Comparison of various levels of Fusion of Multi-focused Images using Wavelet Transform", 2010 International Journal of Computer Applications (0975 8887), Volume 1 - No. 6.

[11] Jiang Dong, Dafang Zhuang, Yaohuan Huang and Jingying Fu, "Advances in Multi-sensor data fusion: algorithm and applications", Sensors 2009, 9, 7771-7784; doi: 10.3390/s91007771.

[12] Vijayaraj, V.; Younan, N. ; O' Hara, “C. Concepts of image fusion in remote sensing applications". In
Proceedings of IEEE International Conference on Geoscience and Remote Sensing Symposium, Denver, CO, USA, July 31 - August 4, 2006; pp. 3798-3801.

[13] Wenzhong Shi, ChangQing Zhu, Yan Tian, Janet Nichol, "Wavelet-based image fusion and quality assessment", International Journal of Applied Earth Observation and Geoinformation 6 (2005) 241-251.

[14] YAO Wan-qiang, ZHANG Chun-sheng, "Multi-spectral image fusion method based on wavelet transformation", The International Archives of the Photogrammetry, Remote Sensing and Spatial Information Sciences. Vol. XXXVII. Part B7. Beijing 2008.

[15] Louis, E.K.; Yan, X.H. "A neural network model for estimating sea surface chlorophyll and sediments from thematic mapper imagery". Remote Sens. Environ. 1998, $66,153-165$.

[16] Dong. J.; Yang, X.; Clinton, N.; Wang, N. “An artificial neural network model for estimating crop yields using remotely sensed information”. Int. J. Remote Sens. 2004, $25,1723-1732$

[17] Shutao, L.; Kwok, J.T.; Yaonan W. "Multifocus image fusion using artificial neural networks", Pattern Recognit. Lett. 2002, 23, 985-997.

[18] Hossein Sahoolizadeh, Davood Sarikhanimoghadam, and Hamid Dehghani, "Face Detection using Gabor Wavelets and Neural Networks", World Academy of Science, Engineering and Technology 45, 2008.

[19] Wang, R.; Bu, F.L.; Jin, H.; Li, L.H. "A feature-level image fusion algorithm based on neural networks". Bioinf. Biomed. Eng. 2007, 7, 821-824.

[20] R.Maruthi, and Dr.K.Sankarasubramanian, "Multi focus image fusion based on the Information level in the regions of the images", Journal of Theoretical and Applied Information Technology, () 2005 - 2007 JATIT.

[21] H.Li, S. Manjunath and S.K. Mitra, "Multi-sensor image fusion using the wavelet transform" in Graphical Models and Image Processing, vol. 57, no.3, pp. 235-245, 1995. 